\title{
Favipiravir: A Possible Pharmaceutical Treatment for COVID-19
}

\author{
Hidekatsu Yanai
}

Severe acute respiratory syndrome coronavirus 2 (SARSCoV-2), which causes coronavirus disease 2019 (COVID-19) has reached a pandemic level. There is an urgent need for effective treatment. Currently, there is not any specific effective antiviral treatment for COVID-19. Supportive care remains the mainstay of therapy for COVID-19. At present, various antiviral and immunomodulating agents are in various stages of evaluation for COVID-19. The frequently used agents all over the world include chloroquine, hydroxychloroquine, lopinavir/ritonavir, favipiravir and remdesivir [1]. SARS-CoV-2 genome-based specific vaccines and therapeutic antibodies are currently being tested; however, we need more time to use such vaccines and antibodies. The existing therapeutic agents previously designed for other virus infections and pathologies attract attention because most of these agents have already been tested for their safety. These agents can be divided into two broad categories, those that can directly target the virus replication, and those based on immunotherapy which improves damage induced by inflammatory responses [2]. The initial clinical studies indicated the promising therapeutic potential of favipiravir, a broad-spectrum antiviral drug that interferes with the viral replication [2]. Here, I describe possible effects of favipiravir for COVID-19.

Favipiravir plays as an inhibitor of the RNA-dependent RNA polymerase by structurally resembling the endogenous guanine [3]. Through competitive inhibition, the efficacy of viral replication can be largely reduced. Favipiravir has been shown to be effective in the treatment of influenza and Ebola virus [3-5]. Very recently, Wang et al showed that favipiravir was effective in reducing the SARS-CoV-2 infection in vitro [6]. In March 2020, favipiravir was approved by the National Medical Products Administration of China as the first anti-COVID-19 drug in China, because the clinical trial had demonstrated efficacy with minimal side effects. Chen et al conducted a prospective, randomized, controlled, openlabel multicenter trial involving adult patients with COVID-19 (ChiCTR2000030254) [7]. Patients were randomly assigned to receive conventional therapy plus favipiravir or arbidol which

Manuscript submitted April 21, 2020, accepted April 27, 2020

Department of Diabetes, Endocrinology and Metabolism, National Center for Global Health and Medicine Kohnodai Hospital, 1-7-1 Kohnodai, Ichikawa, Chiba 272-8516, Japan. Email: dyanai@hospk.ncgm.go.jp

doi: https://doi.org/10.14740/jem645 is a broad-spectrum antiviral compound that blocks viral fusion [8], for 10 days. Two hundred forty enrolled COVID-19 patients underwent randomization; 120 patients were assigned to receive favipiravir, and 120 to receive arbidol. Favipiravir led to shorter latencies to relief for both pyrexia by 1.70 days $(\mathrm{P}<0.0001)$ and cough by 1.75 days $(\mathrm{P}<0.0001)$. However, no difference was observed of the rate of auxiliary oxygen therapy or noninvasive mechanical ventilation. The most frequently observed favipiravir-associated adverse event was elevation of serum uric acid.

Cai et al performed an open-label control study to evaluate experimental treatment with favipiravir for COVID-19 [9]. Patients were assigned to receive oral favipiravir plus interferon- $\alpha$ aerosol inhalation ( $\mathrm{n}=35)$, or receive lopinavir/ ritonavir which is a fixed-dose protease inhibitor combination used for the treatment of human immunodeficiency virus 1 (HIV-1) [10] plus interferon- $\alpha$ aerosol inhalation $(n=45)$. A viral clearance time was significantly shorter in the favipiravir arm than the lopinavir/ritonavir arm by 7 days (median value). Further, multivariable Cox regression showed that favipiravir was independently associated with faster viral clearance. The favipiravir arm also showed significant improvement in chest imaging compared with the lopinavir/ritonavir arm, with an improvement rate of $91.43 \%$ versus $62.22 \%$ at day 4 after treatment. Total number of adverse reactions in the favipiravir group $(\mathrm{n}=4,11.4 \%)$ was significantly smaller than the lopinavir/ritonavir group $(n=25,55.56 \%)$. Nausea was significantly more frequent in the lopinavir/ritonavir group than the favipiravir group, and vomiting tended to be frequent in the lopinavir/ritonavir group than the favipiravir group.

The Japanese Association for Infectious Diseases reported an elderly patient (Ishikawa et al) or a hemodialysis patient (Saito et al), whose COVID-19 pneumonia was alleviated by favipiravir as the case reports on their homepage [11]. The Japanese Association for Infectious Diseases also reported that an improvement was observed in $90 \%, 85 \%$ and $61 \%$, after 14 days from the start of favipiravir, in mild, moderate and severe COVID-19 cases, respectively, on their web symposium.

Favipiravir may be a relatively safe and effective drug for COVID-19 at present. We have to work together to combat and win against COVID-19, regardless of our medical specialty. We will get a great victory against COVID-19 in the near future.

\section{Acknowledgments}

None to declare. 


\section{Financial Disclosure}

None to declare.

\section{Conflict of Interest}

The author declares that he has no conflict of interest concerning this article.

\section{Author Contributions}

HY collected literatures and wrote and approved the final paper.

\section{Data Availability}

The author declares that data supporting the findings of this study are available within the article.

\section{References}

1. Simsek Yavuz S, Unal S. Antiviral treatment of COVID-19. Turk J Med Sci. 2020;50(SI-1):611-619.

2. Tu YF, Chien CS, Yarmishyn AA, Lin YY, Luo YH, Lin
YT, Lai WY, et al. A Review of SARS-CoV-2 and the Ongoing Clinical Trials. Int J Mol Sci. 2020;21(7):2657.

3. Furuta Y, Gowen BB, Takahashi K, Shiraki K, Smee DF, Barnard DL. Favipiravir (T-705), a novel viral RNA polymerase inhibitor. Antiviral Res. 2013;100(2):446-454.

4. Sissoko D, Laouenan C, Folkesson E, M'Lebing AB, Beavogui AH, Baize S, Camara AM, et al. Experimental treatment with favipiravir for ebola virus disease (the JIKI Trial): a historically controlled, single-arm proof-ofconcept trial in guinea. PLoS Med. 2016;13(3):e1001967.

5. Bouazza N, Treluyer JM, Foissac F, Mentre F, Taburet AM, Guedj J, Anglaret X, et al. Favipiravir for children with Ebola. Lancet. 2015;385(9968):603-604.

6. Wang M, Cao R, Zhang L, Yang X, Liu J, Xu M, Shi Z, et al. Remdesivir and chloroquine effectively inhibit the recently emerged novel coronavirus $(2019-\mathrm{nCoV})$ in vitro. Cell Res. 2020;30(3):269-271.

7. Chen C, Zhang Y, Huang J, Yin P, Cheng Z, Wu J, Chen S. Favipiravir versus Arbidol for COVID-19: a randomized clinical trial. medRxiv 2020:2020.03.17.20037432.

8. Boriskin YS, Leneva IA, Pecheur EI, Polyak SJ. Arbidol: a broad-spectrum antiviral compound that blocks viral fusion. Curr Med Chem. 2008;15(10):997-1005.

9. Cai Q, Yang M, Liu D, Chen J, Shu D, Xia J, et al. Experimental treatment with favipiravir for COVID19: an open-label control study. Engineering. 2020. In press.

10. Corbett AH, Lim ML, Kashuba AD. Kaletra (lopinavir/ ritonavir). Ann Pharmacother. 2002;36(7-8):1193-1203.

11. http://www.kansensho.or.jp. 